\title{
Christmas connections
}

\section{Emer McLoughlin, Helen Williams}

This collection of cases has a Yuletide theme; think winter weather and the nativity story with a dose of lateral thinking to link the imaging findings with a Christmas themed paediatric radiology sign.

Answers to the questions can be found on page 291

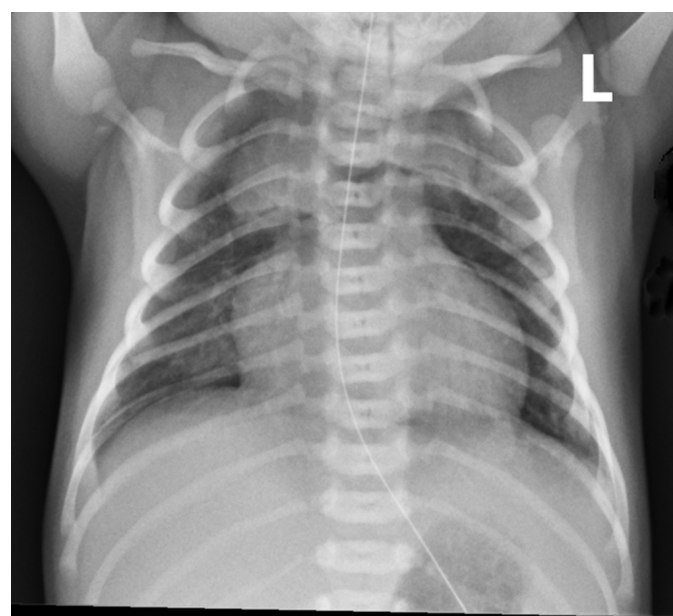

Figure 1

\section{QUESTION 1}

A chest X-ray (figure 1) was taken to assess this term baby with respiratory distress at 4 hours of age. What celestial sign is demonstrated and why does it occur?

A

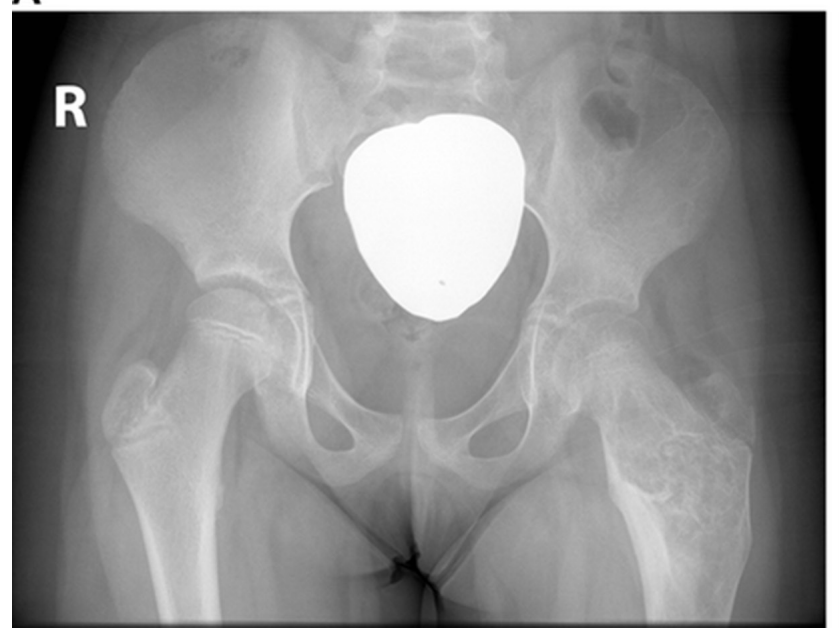

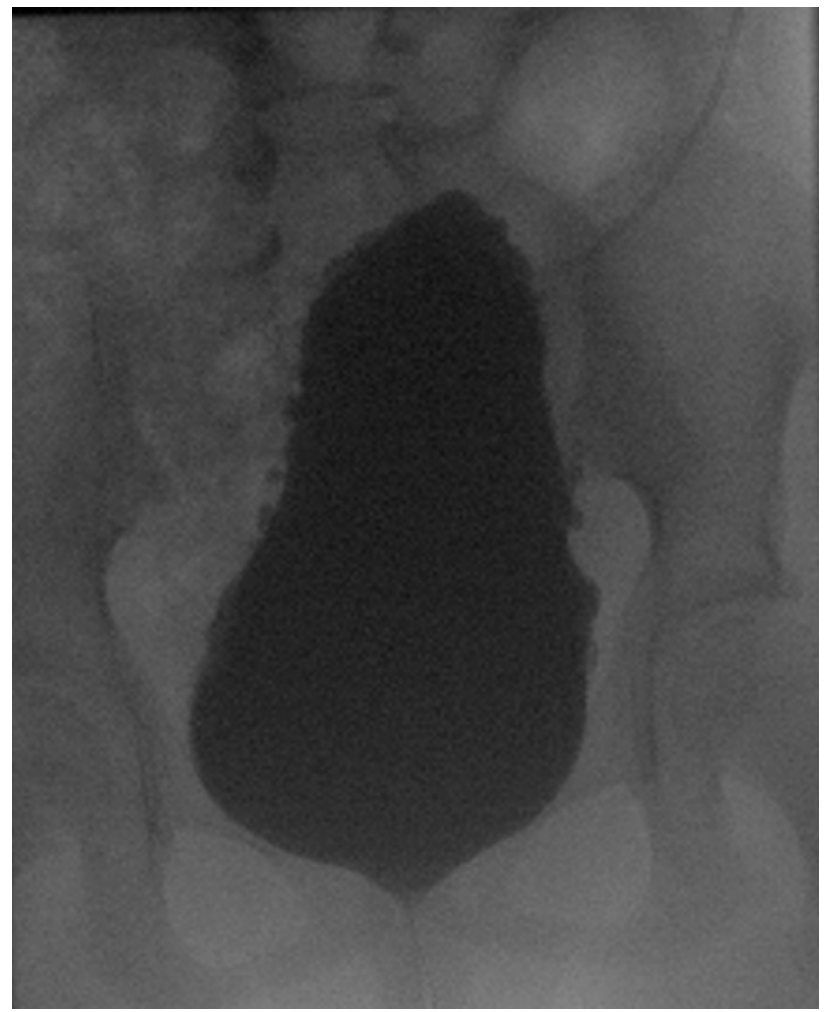

Figure 2

\section{QUESTION 2}

What evergreen entity is seen on this cystogram image (figure 2) taken during urodynamic bladder assessment of a an 11-year old girl with spina bifida? What causes it?

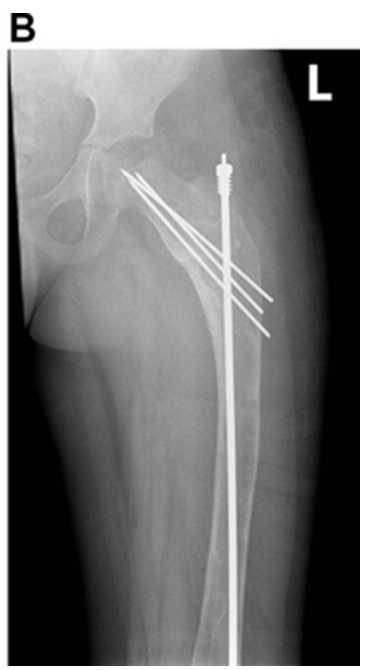

Figure 3

\section{QUESTION 3}

An 8-year old girl known to the paediatric orthopaedic surgeons presented to the emergency department with leg pain and subsequently had surgery to stabilise her left femur. Her preoperative and postoperative radiographs are shown in figure $3 \mathrm{a}$ and figure $3 \mathrm{~b}$. What curved appendage linked with the nativity shares its name with the bowed appearance of this patient's left proximal femur? What is the underlying diagnosis in her case? 


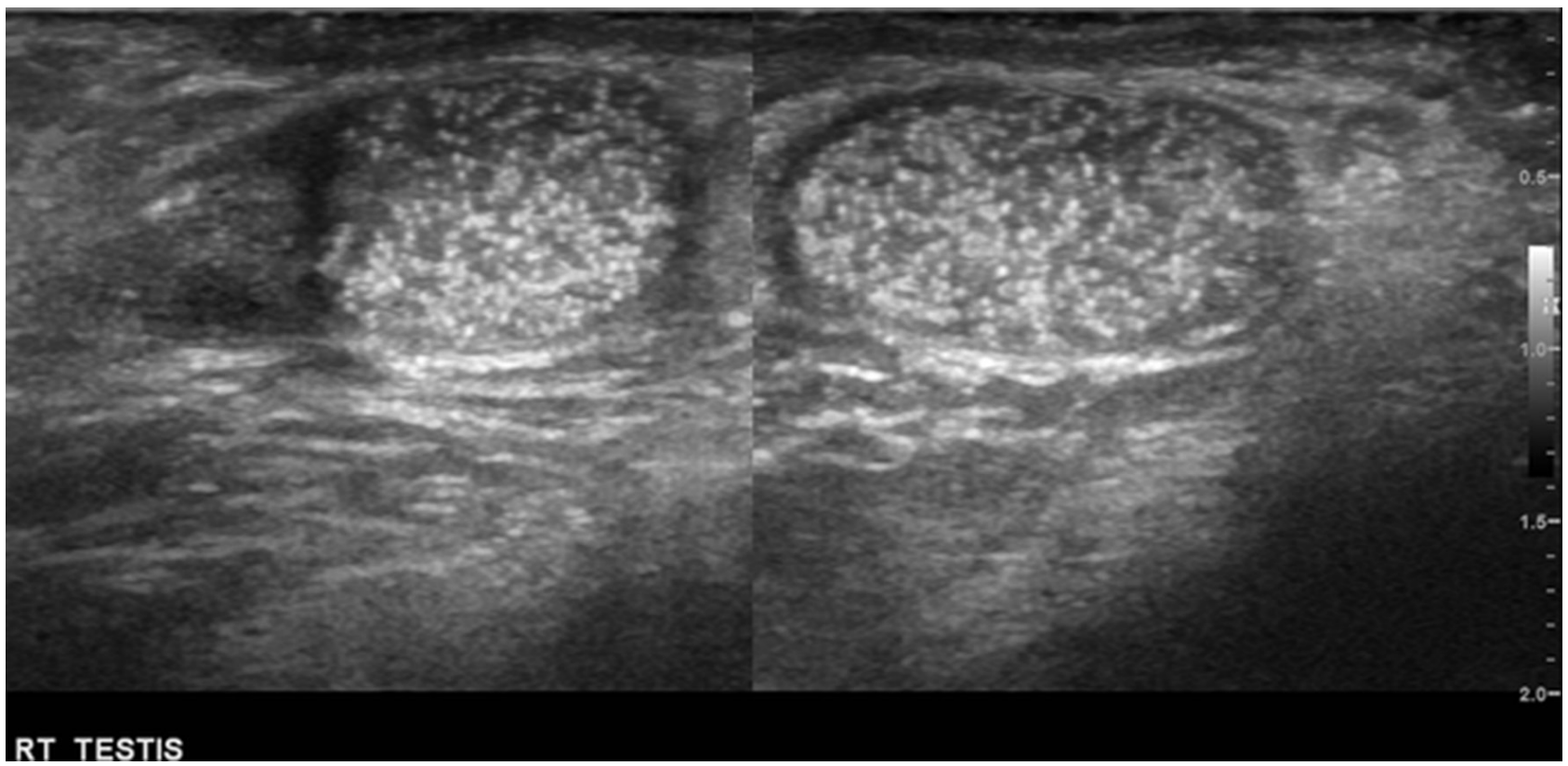

Figure 4

\section{QUESTION 4}

This precipitous presentation was found on ultrasound in an 8-year old boy with undescended testes. Both testicles were in the inguinal canal and had similar appearances. Ultrasound of the right testicle is shown in figure 4 . What is the name of the sign causing this blizzard appearance? What is its significance in children?

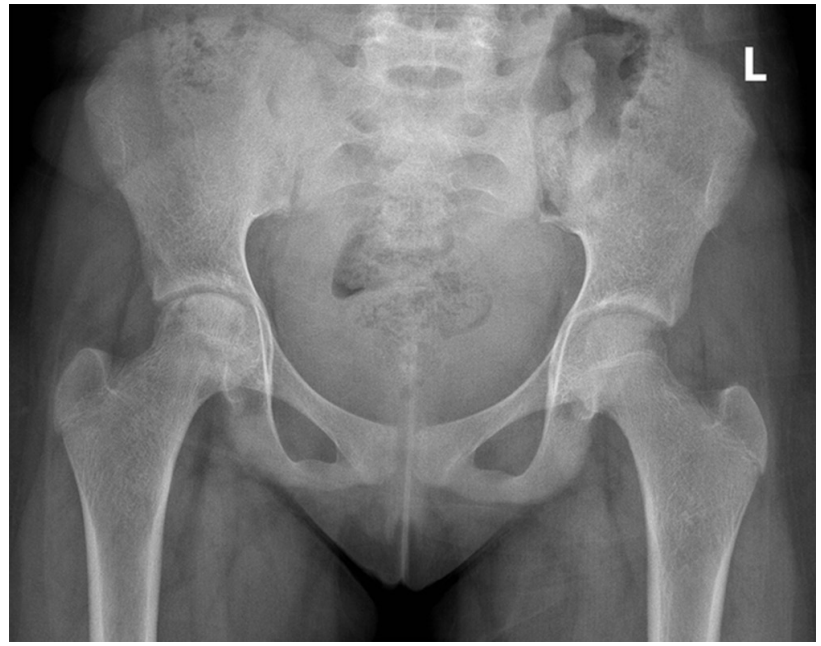

Figure 5

\section{QUESTION 5}

The pelvic radiograph shown in figure 5 was taken to assess the hips of a 15 -year old girl with sickle cell disease. She presented with a limp associated with right hip pain for several weeks. What alpine adjective describes the appearance of the right femoral head and what is the cause?

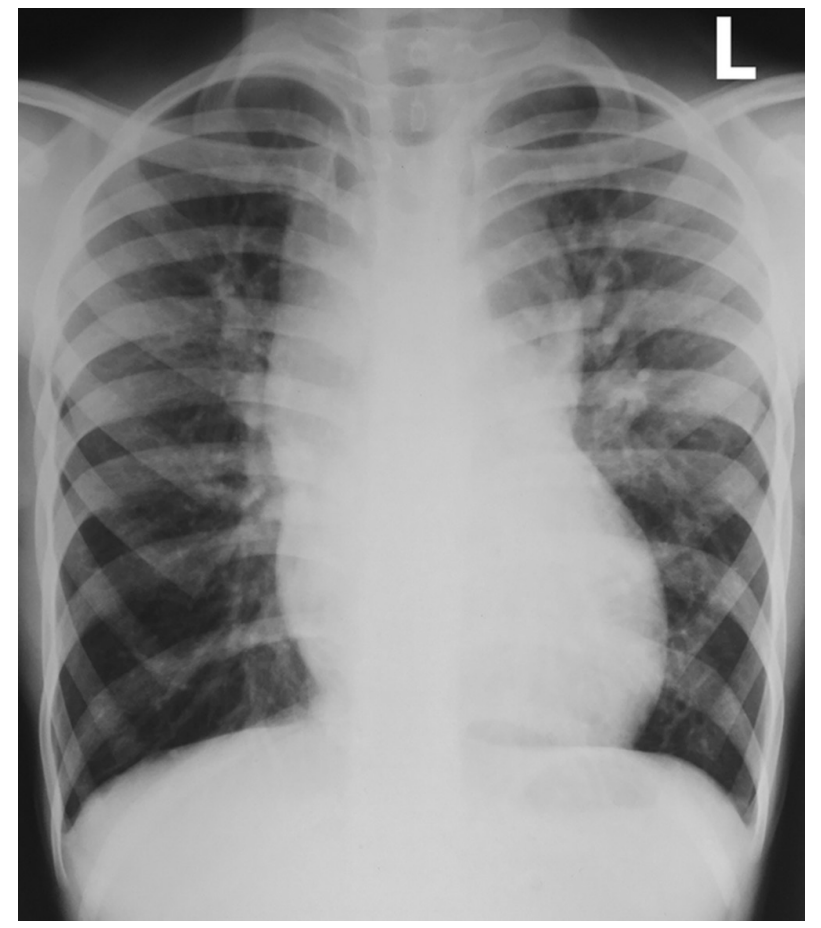

Figure 6

\section{QUESTION 6}

The chest X-ray shown in figure 6 is of an 8-year old girl who was found to have low oxygen saturations (85\%) during routine postoperative monitoring. A systolic flow murmur was detected on examination. What snowy sign is demonstrated and why is the mediastinum this shape? 
ANSWERS TO THE QUESTIONS ON PAGE 289 AND 290

\section{ANSWER TO QUESTION 1}

The chest X-ray shows the 'angel wings' sign of pneumomediastinum in a neonate. Both lobes of the thymus are elevated by air in the mediastinum producing this sign, which is sometimes referred to as the spinnaker sign. Spontaneous neonatal pneumothorax is associated with aspiration of blood or meconium and birth-related trauma, but can also result from positive pressure ventilation. In term babies it is generally asymptomatic, but is occasionally accompanied by mild tachypnoea. Rarely, pneumomediastinum is associated with an oxygen requirement or develops into pneumothorax.

\section{ANSWER TO QUESTION 2}

The cystogram image demonstrates an elongated urinary bladder with tapering towards the bladder dome giving a conical appearance which resembles a Christmas tree. 'Christmas tree' bladder describes the radiological appearance of a severely neurogenic bladder. The bladder wall is thickened and trabeculated and the subsequent irregular contour gives the appearance of decorations hanging from a Christmas tree.

Neurogenic bladder is defined as urinary bladder malfunction due to neurologic dysfunction. In children, the most common cause of a neurogenic bladder is a congenital sacral abnormality such as spina bifida. Damage to the central or peripheral nervous system from infection, trauma, malignancy or vascular insult may also result in a dysfunctional bladder.

\section{ANSWER TO QUESTION 3}

The radiographs demonstrate an abnormal contour of the proximal femur, with bony expansion and abnormal angulation, known as 'shepherd's crook' deformity. This is the classical appearance of the proximal femur in the setting of fibrous dysplasia, although this type of deformity can also be caused by other bony conditions such as osteogenesis imperfecta.

Fibrous dysplasia is a non-neoplastic tumourlike disorder in which normal bone and marrow are replaced with fibrous tissue. This results in weakening of the affected bone leading to deformity or fracture. Fibrous dysplasia can affect a single bone (monostotic) or multiple bones (polyostotic) and is found predominantly in children and young adults, with the majority presenting before 30 years of age. The lesions do not progress after puberty and surgical intervention may only be required if there is pathological fracture, severe deformity or significant mass effect on surrounding structures.

\section{ANSWER TO QUESTION 4}

The testicular ultrasound demonstrates the 'snowstorm' sign of testicular microlithiasis (TM). Multiple tiny, hyperechoic, calcific foci, without acoustic shadowing are present within the testes giving a 'snowstorm' appearance. The ultrasound diagnosis can only be made if more than five calcific foci are identified. In this patient, the testes are abnormally small and the calcifications are profuse.

Testicular microlithiasis is a relatively common condition with a reported incidence of $2 \%$ in the paediatric population. The clinical relevance of TM arises from its potential link with testicular tumours, both benign and malignant. A significant association between TM and testicular tumours has been reported with an incidence of $12 \%$ in boys with TM. However, most boys with TM and a testicular tumour have an underlying predisposition for a testicular tumour (eg, undescended testes or Peutz-Jeghers syndrome) and therefore, the benefit of screening boys with TM remains uncertain.

\section{ANSWER TO QUESTION 5}

The pelvic radiograph demonstrates a 'snow-capped' appearance of the right femoral head seen in avascular necrosis (AVN). AVN is death of bone constituents secondary to disturbance of blood supply. The 'snowcap' sign is used to describe the dense sclerosis of the femoral head which occurs during the healing phase of avascular necrosis, when revascularisation occurs.

Avascular necrosis of the femoral head is a common complication in patients with sickle cell disease. Other risk factors for developing AVN include corticosteroid use, Gaucher's disease, chemotherapy and radiotherapy and trauma. Idiopathic avascular necrosis of the femoral head in children is known as Perthe's disease which is a diagnosis of exclusion in the absence of other causes of AVN. There is a male predilection for Perthe's disease and it is more common in the 5-7years age group. Children with AVN of the femoral head most commonly present with atraumatic hip pain or a limp.

\section{ANSWER TO QUESTION 6}

The chest radiograph demonstrates an abnormal configuration of the heart and the superior mediastinum resembling a snowman which is seen in the supracardiac variant of total anomalous pulmonary venous return (TAPVR). There is cardiomegaly, increased pulmonary arterial markings and widening of the superior mediastinum.

TAPVR is an congenital abnormality of the circulation in which the pulmonary veins drain into the right atrium or its venous tributaries rather than the left atrium. Communication between the atria (an atrial septal defect or patent foramen ovale) is necessary for survival. In supracardiac TAPVR, the pulmonary veins converge to form a common pulmonary vein which joins with a left-sided vertical vein. The vertical vein connects with the brachiocephalic vein which drains into the SVC. Widening of the superior 
mediastinum is caused by the prominent SVC on the right and the vertical vein on the left. The brachiocephalic vein crosses the midline in the superior mediastinum and these vessels together shape the snowman's head. The snowman's body is formed by the heart and enlarged right cardiac chambers. This distinctive appearance is also known as the 'figure of 8 ' or 'cottage loaf' sign.

In childhood, unobstructed supracardiac TAPVR typically presents with failure to thrive, frequent chest infections or signs of congestive heart failure. However, patients may be asymptomatic with incidental finding of a murmur, an abnormal chest radiograph or relatively mild systemic arterial desaturation which is not clinically detectable.
Correspondence to Dr Helen Williams, Department of Radiology, Birmingham Children's Hospital, Steelhouse Lane, Birmingham B4 6NH, UK; helen.williams32@nhs.net

Competing interests None delared.

Provenance and peer review Commissioned; internally peer reviewed.

(c) Article author(s) (or their employer(s) unless otherwise stated in the text of the article) 2017. All rights reserved. No commercial use is permitted unless otherwise expressly granted.

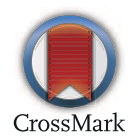

To cite McLoughlin E, Williams H. Arch Dis Child Educ Pract Ed 2017;102:289-292.

Accepted 17 July 2017 